ARTIGO DE REVISÃO

\title{
Comunidade de prática na formação e desenvolvimento profissional na educação física: uma metassíntese
}

\author{
Community of practice in physical education teacher education and professional development: \\ a metasynthesis
}

\author{
Leonardo Ristow ${ }^{1}$, Ana Flávia Backes², Daniela Bianchessi ${ }^{3}$, Vinicius Zeilmann Brasil ${ }^{1}$, \\ Rodolfo Silva da Rosa ${ }^{1}$, Jessica Cardoso ${ }^{1}$, Valmor Ramos ${ }^{1}$ \\ ${ }^{1}$ Universidade do Estado de Santa Catarina (UDESC), Florianópolis/SC, Brasil \\ 2 Universidade Federal de Santa Catarina (UFSC), Florianópolis/SC, Brasil \\ ${ }^{3}$ Centro Universitário de Brusque (Unifebe), Brusque/SC, Brasil
}

\section{HISTÓRICO DO ARTIGO \\ Recebido: 13 julho 2020 \\ Revisado: 22 outubro 2020 \\ Aprovado: 23 outubro 2020}

\section{PALAVRAS-CHAVE:}

Educação Física; Comunidade de prática; Formação e

desenvolvimento profissional.

\section{KEYWORDS:}

Physical Education; Community of practice; Teacher education and professional development.

\section{RESUMO}

INTRODUÇÃO: A aprendizagem e o desenvolvimento profissional em Educação Física ocorrem em diferentes contextos. Entre eles, as comunidades de prática são consideradas um contexto promissor, pois possibilitam a participação ativa, envolvendo os membros em um ambiente colaborativo onde o aprendizado e o desenvolvimento ocorrem com e através das relações pessoais.

OBJETIVO: Desse modo, o objetivo deste estudo foi realizar uma metassíntese a respeito dos fatores que influenciam o desenvolvimento e manutenção das comunidades de prática de professores e futuros professores de Educação Física.

MÉTODOS: A metassíntese é uma metodologia de revisão sistemática que sintetiza os resultados de estudos qualitativos em categorias. Os resultados dos estudos originais foram analisados e sintetizados a partir da análise de conteúdo. As categorias foram determinadas a priori: engajamento mútuo, empreendimento em conjunto e repertório compartilhado.

RESULTADOS: Os resultados dos 16 estudos selecionados nos indicam que as comunidades de prática na formação e no desenvolvimento profissional tem origem a partir das necessidades dos interessados. A negociação quanto ao tema ou conteúdo dos encontros ocorreram tanto no início quanto ao longo dos encontros. A comunidades de prática apresentaram diferentes objetivos: aprender a ensinar Educação Física, aprender a implementar modelos de ensino, elaborar um novo currículo, aprender a aplicar uma determinada abordagem de ensino e aperfeiçoar práticas de ensino. Já a manutenção ocorreu a partir das trocas de experiências profissionais e das relações pessoais informais em diferentes contextos: em redes sociais, no estágio obrigatório do curso de graduação, na disciplina do curso de graduação e encontro de grupos de professores. Vale destacar o papel do facilitador para que o repertório compartilhado fosse criado.

CONCLUSÃO: Em suma, as comunidades de prática se apresentam como um excelente contexto de aprendizagem e desenvolvimento profissional, já que as comunidades de prática investigadas parecem ter satisfeito as necessidades dos participantes.

\section{ABSTRACT}

BACKGROUND: Learning and professional development in Physical Education occur in different contexts. Among them, communities of practice are considered to be a promising context as they enable active participation. Engaging members in a collaborative environment where learning and development take place with and through personal relationships.

OBJECTIVE: Thus, the aim of this study was to carry out a meta-synthesis about the factors that influence the development and maintenance of communities of practice for teachers and future Physical Education teachers. METHODS: Metasynthesis is a systematic review methodology that synthesizes the results of qualitative studies in categories. The results of the original studies were analyzed and synthesized from the content analysis. The categories were determined a priori: mutual engagement, joint enterprise and shared repertoire.

RESULTS: The results of the 16 selected studies indicate that the communities of practice in teacher education and professional development originate from the needs of those interested. Negotiations regarding the theme or content of the meetings took place both at the beginning and throughout the meetings. The communities of practice had different objectives: learn to teach Physical Education, learn to implement teaching models, develop a new curriculum, learn to apply a particular teaching approach and improve teaching practices. Maintenance, on the other hand, occurred based on the exchange of professional experiences and informal personal relationships in different contexts: in social networks, in the mandatory stage of the undergraduate course, in the subject of the undergraduate course and meeting of groups of teachers. It is worth highlighting the role of the facilitator so that the shared repertoire was created.

CONCLUSION: In short, communities of practice present themselves as an excellent context for learning and professional development, since the communities of practice investigated seem to have satisfied the needs of the participants. 


\section{INTRODUÇÃO}

A aprendizagem profissional em Educação Física ocorre em diferentes contextos. Ao longo da formação inicial e posteriormente na carreira docente, as habilidades de ensino se desenvolvem por meio de experiências sociais, seja com professores formadores, colegas da graduação ou colegas de profissão (FLORES et al., 2019; GOMES et al., 2014; EIRÍNNEMIÑA, 2018). Nos últimos anos, estudos têm como foco investigar como os professores aprendem através dessas experiências sociais e como se organizam os grupos ou comunidades de professores (HUNUK; INCE; TANNEHILL, 2018).

Diferente das formas tradicionais de formação e capacitação profissional, em que os participantes atuam como meros receptores de informações, as comunidades de prática, envolvem os professores em um ambiente de colaboração, aprendendo com e através das relações sociais (PATTON; PARKER; PRATT, 2013; PARKER; PATTON; TANNEHILL, 2012). De fato, acredita-se que a prática docente se altera quando os professores participam ativamente do processo de aprendizagem profissional, focado em suas próprias necessidades (GOODYEAR; CASEY, 2015; TANNEHILL; MacPHAIL, 2017; HUNUK; TANNEHILL; INCE, 2019).

Dentre as diferentes formas de aprendizado social e participativa (VYGOSTKY, 1991), as comunidades de prática têm sido consideradas uma estrutura promissora na formação de futuros professores (GOMES et al., 2014; CARDOSO; BATISTA; GRAÇA, 2016; YOUNG; MacPHAIL, 2017) e no desenvolvimento profissional de professores de Educação Física (GOODYEAR; CASEY; KIRK, 2014; YOON; ARMOUR, 2017; LUGUETTI et al., 2018). As comunidades de prática são descritas por Wenger (2013, p. 1, tradução nossa) como "grupos de pessoas que compartilham uma preocupação ou uma paixão por algo que fazem e aprendem a fazê-lo melhor à medida que interagem regularmente".

Apesar das investigações a respeito das comunidades de prática no âmbito da formação e desenvolvimento profissional em Educação Física têm evidenciado benefícios, uma preocupação eminente para pesquisadores e professores formadores se refere ao entendimento de como os professores são influenciados a participar das CdP e como essas comunidades são implementadas (GOODYEAR; CASEY, 2015). Diante do exposto, o objetivo deste estudo foi realizar uma metassíntese a respeito dos fatores que influenciam o desenvolvimento e manutenção das comunidades de prática de professores e futuros professores de Educação Física.

\section{MÉTODOS}

Foi realizada uma metassíntese, que é caracterizada como uma metodologia de revisão sistemática que sintetiza os resultados de estudos qualitativos em categorias (SANDELOWSKI; BARROSO, 2006). Esse tipo de revisão permite a análise e a integração de resultados sobre determinado tema de investigação. Trata-se, da interpretação dos pesquisadores sobre os dados primários dos estudos originais. Assim, a metassíntese qualitativa tem como objetivo apresentar interpretações ampliadas de todos os estudos selecionados sobre uma determinada temática, respeitando a tradução interpretativa de cada estudo (ZIMMER, 2006).
Para manter o rigor e a clareza no processo de identificação dos artigos, a seleção foi realizada com base nos procedimentos indicados pelo fluxograma Preferred Reporting Intems for Systematic Reviews and Meta-Analysis (PRISMA). Este tipo de estudo é constituído por três etapas: revisão sistemática, análise crítica dos artigos e síntese dos resultados.

\section{Etapa 1: Revisão sistemática}

Na primeira fase, foi realizada uma busca na base de dados Periódicos da Capes, entre os dias 20 e 22 de janeiro de 2020. Foram selecionados apenas os artigos revisados por pares e publicados na íntegra entre 2010 e 2019. Para a identificação dos estudos, foram utilizadas duas sintaxes: "Communities of practice" AND "Physical Education" sendo encontrados 86 artigos e, "Community of practice" AND "Physical Education" sendo encontrados 46 artigos. Dos 132 artigos selecionados, 32 foram excluídos por duplicidade. Ao final da etapa de identificação, foram encontrados 100 artigos.

Na segunda fase, para a seleção dos artigos dois pesquisadores, individualmente, analisaram o título e o resumo de cada artigo, para certificar que estes atendiam aos critérios de inclusão: ter o texto disponível na íntegra, ser um estudo empírico de abordagem qualitativa, ter como contexto investigado uma ou mais comunidades de prática (WENGER, 1998) que tinham como foco a prática pedagógica de professores e futuros professores de Educação Física. Quando houve divergências entre os pesquisadores referente à seleção, um terceiro pesquisador tomou a decisão final. Nesta etapa, foram excluídos 36 artigos.

$\mathrm{Na}$ última fase referente a elegibilidade, os 64 estudos foram analisados na íntegra com o intuito de verificar se estes atendiam aos critérios de inclusão supracitados. Ao final desse processo, 48 artigos foram excluídos e 16 foram selecionados para a análise crítica. Destes, foram analisadas as referências e nenhum artigo foi incluído manualmente. Os procedimentos de identificação, seleção e elegibilidade pelos quais incluíram os artigos para a metassíntese é ilustrado no fluxograma (Figura 1).

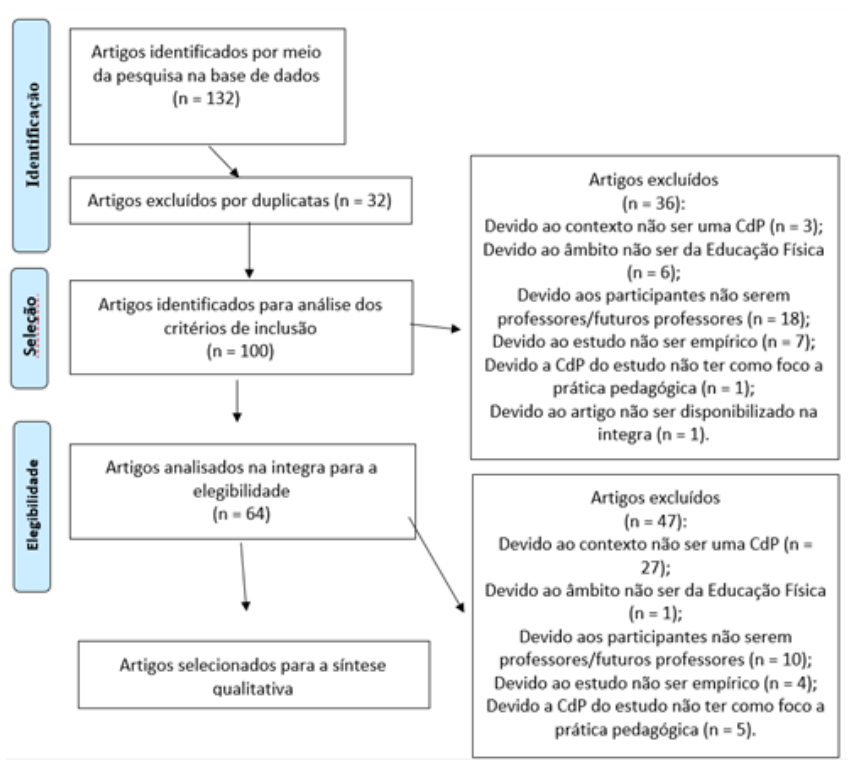

Figura 1. Fluxograma da seleção dos artigos. 


\section{Etapa 2: Análise crítica}

Nesta etapa, dois pesquisadores, individualmente, analisaram na íntegra os 16 artigos selecionados e realizaram a avaliação crítica dos artigos com base no Critical Appraisal Skills Programme (CASP) para pesquisas qualitativas, um instrumento que descreve as diretrizes para a análise de qualidade das pesquisas qualitativas. Para o presente estudo, a análise da qualidade não foi usada como instrumento de seleção, pois de acordo com Sandelowski e Barroso (2006), a exclusão de estudos considerando apenas os critérios de qualidade, podem desperdiçar informações importantes a respeito do objeto de estudo.

Quando houve divergências nas análises dos pesquisadores, um terceiro pesquisador tomou a decisão a respeito da qualidade do artigo. O CASP possui dez itens que classificam os estudos em (A) baixo risco de viés e (B) risco de viés moderado. Os itens analisados são: objetivo claro e justificado; desenho metodológico apropriado aos objetivos; procedimentos metodológicos apresentados e discutidos; seleção da amostra intencional; coleta de dados descrita, instrumentos explicitados e processo de saturação; relação entre pesquisador e pesquisado; cuidados éticos; análise densa e fundamentada; resultados apresentados e discutidos, apontando o aspecto da credibilidade e uso da triangulação; descrição sobre as contribuições e implicações do conhecimento gerado pela pesquisa.

\section{Síntese dos resultados}

Os resultados dos 16 estudos originais foram analisados e sintetizados a partir da análise de conteúdo, que segundo Bardin (2016, p. 44), é um "conjunto de técnicas de análise das comunicações utilizadas para explicitar e sistematizar o conteúdo das mensagens". As categorias foram determinadas a priori de acordo com a Teoria Social da Aprendizagem (WENGER, 1998): Empreendimento em Conjunto, que diz respeito ao compartiIhamento de ideias e da participação em discussões, reflexões e soluções de problemas em conjunto. Nem sempre essa relação acontece de modo pacífico, visto a complexidade de tais reflexões e ações; Engajamento Mútuo que é caracterizado como um processo coletivo de negociação entre as ideias discutidas por parte do engajamento mútuo, reforçando a importância das reflexões sugeridas pelos participantes; e Repertório Compartilhado que se refere a produção e/ou sistematização dos conceitos, palavras, ações, ferramentas, estratégias e práticas adotadas pela $\mathrm{CdP}$, que são construídas a partir das discussões formadas nas duas dimensões anteriores, facilitando o andamento e desenvolvimento das interações que são construídas por meio da CdP (ELLERY; PONTES, LOIOLA, 2012). Esta etapa foi realizada em reunião de três pesquisadores. A organização dos artigos e a sistematização dos resultados foi realizada com o auxílio de uma planilha no software Microsoft Office Excel (Versão 2010).

\section{RESULTADOS}

Todos os estudos selecionados apresentam baixo risco de viés de acordo com o CASP. Referente ao procedimento metodológico, apenas um utilizou método de pesquisa misto, porém para o presente estudo foram considerados apenas os dados qualitativos. Quanto aos instrumentos utilizados, observa-se a utilização de uma diversidade de fontes de dados: entrevistas individuais, entrevistas informais, entrevista com grupos focais, diário reflexivo, questionários, escrita em redes sociais, anotações de campo, filmagem das aulas práticas, filmagem dos encontros, planos de aula (artefatos), entre outros (Quadro 1).

\section{Empreendimento em conjunto}

As comunidades de prática investigadas nos estudos selecionados apresentaram diferentes focos, como: aprender a ensinar Educação Física (GOMES et al., 2014; CARDOSO; BATISTA; GRAÇA, 2016; YOUNG; MacPHAIL, 2015); aprender a implementar modelos de ensino (GOODYEAR; CASEY; KIRK, 2014; YOON; ARMOUR, 2017; LUGUETTI et al., 2019); elaborar um currículo (PARKER et al., 2010); aprender a aplicar determinada abordagem de ensino (HUNUK; INCE; TANNEHILL, 2013; OLIVER et al., 2018; LUGUETTI et al., 2019); e aperfeiçoar práticas de ensino (PARKER; PATTON; TANNEHILL, 2012; PATTON; PARKER; PRATT, 2013; GOODYEAR; CASEY, 2015; MARTIN, 2017; PATTON; PARKER, 2017; EIRÍN-NEMIÑA, 2018).

A participação em uma comunidade de prática, de acordo com Bertram, Culver e Gilbert (2017) auxilia no desenvolvimento das concepções de educação, bem como metodologias e estratégias de ensino. Por meio da participação efetiva, é possível refletir e guiar os estudos de uma comunidade de prática a partir da ideia de formar profissionais crítico-reflexivos sobre sua prática profissional de acordo com as mudanças que ocorrem ao longo do tempo. Assim, para que uma CdP consiga relacionar a comunidade e a prática é preciso um objetivo em comum negociado pelos participantes (WENGER, 1998).

Nos estudos selecionados, a negociação coletiva a respeito do objetivo da comunidade foi determinada de diferentes maneiras: Antes da criação da CdP (no caso de aprender a usar determinado modelo e abordagem de ensino, assim como para elaborar o currículo), como se observa no trecho de entrevista do estudo de Yoon e Armour (2017, p. 8, tradução nossa): "No início de cada semestre, cada professor trazia seus planos anuais de ensino para compartilhar e discutir uns com os outros. Essas reuniões nos permitiram compartilhar nossas ideias de ensino e aprender umas com as outras". E a partir das necessidades e problemas apresentados pelos participantes (no caso aprender a ensinar Educação Física e aperfeiçoar práticas de ensino), como se observa no trecho de entrevista do estudo de Parker, Patton e Tannehill (2012, p. 319, tradução nossa): "Eu não estaria interessado em ir a uma capacitação ou treinamento, a menos que fosse relevante para o que estava fazendo; que eu poderia voltar e usá-lo. Caso contrário, seria apenas, eu poderia dar um aspecto social, mas tem que ser na verdade o que você está trabalhando e que você pode colocar em prática imediatamente".

Independente do momento em que foi negociado o objetivo da CdP, destaca-se que o interesse em participar deve ocorrer de forma espontânea. Embora deve-se estabelecer um empreendimento em conjunto, cada participante possui um interesse em particular, conforme relatos do estudo de Patton e Parker (2017, p. 354-5, tradução nossa) "alguém com quem conversar e trabalhar", "dar nova vida a mim, me dá paixão e entusiasmo para tentar algo novo e tentar algo um pouco diferente", e "nós apenas sobrevivemos sozinhos. Juntos, podemos reunir conhecimentos, novos conhecimentos. [...] eu tenho muitas novidades em conhecimento que eu nunca esperava antes". 
Quadro 1. Descrição dos autores, participantes, métodos, instrumentos e nível de qualidade dos artigos (CASP) dos estudos selecionados.

\begin{tabular}{|c|c|c|c|c|}
\hline Autor (ano) & Participantes & Método & Instrumentos/coleta de dados & CASP \\
\hline Parker et al. (2010) & 4 professores e 3 facilitadores & Qualitativo/ longitudinal & Entrevistas, anotações de campo e artefatos & A \\
\hline $\begin{array}{l}\text { Parker; Patton; Tannehill } \\
\text { (2012) }\end{array}$ & 33 professores e 7 facilitadores & Qualitativo/ transversal & Entrevistas, anotações de campo, artefatos e entrevistas informais & A \\
\hline $\begin{array}{l}\text { Patton; Parker; Pratt } \\
\text { (2013) }\end{array}$ & $\begin{array}{l}88 \text { professores e } 15 \text { facilita- } \\
\text { dores }\end{array}$ & Qualitativo/ transversal & $\begin{array}{l}\text { Entrevistas semiestruturadas com grupos focais e individuais, entrevistas } \\
\text { informais e artefatos }\end{array}$ & A \\
\hline $\begin{array}{l}\text { Hunuk; Ince; Tennehill } \\
\text { (2013) }\end{array}$ & $\begin{array}{l}12 \text { professores, } 278 \text { estudantes } \\
\text { e } 1 \text { facilitador }\end{array}$ & $\begin{array}{l}\text { Misto/Quase-experi- } \\
\text { mental }\end{array}$ & $\begin{array}{l}\text { Teste de conhecimento de aptidão para professores, Teste de conheci- } \\
\text { mento de aptidão para alunos do ensino médio, entrevistas semiestru- } \\
\text { turadas, notas de campo }\end{array}$ & A \\
\hline Gomes et al. (2014) & $\begin{array}{l}1 \text { professor supervisor, } 1 \text { pro- } \\
\text { fessor e } 3 \text { futuros professores }\end{array}$ & Qualitativo - transversal & $\begin{array}{l}\text { Observação não participante, incluindo anotações de campo, filmagem } \\
\text { das práticas diárias e entrevistas. }\end{array}$ & A \\
\hline $\begin{array}{l}\text { Goodyear; Casey; Kirk } \\
\text { (2014) }\end{array}$ & 6 professores e 1 facilitadora & $\begin{array}{l}\text { Pesquisa-ação partici- } \\
\text { pativa }\end{array}$ & Textos nas redes sociais & A \\
\hline Young; MacPhail (2015) & 18 professores & Qualitativo/ longitudinal & $\begin{array}{l}\text { Diário reflexivo, observações, entrevista semiestruturada e entrevista } \\
\text { em grupo focal. }\end{array}$ & A \\
\hline Goodyear; Casey (2015) & 6 professores e 1 facilitadora & $\begin{array}{l}\text { Pesquisa-ação partici- } \\
\text { pativa }\end{array}$ & $\begin{array}{l}\text { Diário reflexivos, diário de campo, entrevistas, observações e textos nas } \\
\text { redes sociais. }\end{array}$ & A \\
\hline $\begin{array}{l}\text { Cardoso; Batista; Graça } \\
\text { (2016) }\end{array}$ & 1 professor supervisor & Autoetnografia & Diário de bordo da professora & A \\
\hline Martin (2017) & $\begin{array}{l}36 \text { pessoas (criadores da plata- } \\
\text { forma, pesquisadores partici- } \\
\text { pantes, alunos, professores). }\end{array}$ & Estudo de caso & Entrevistas, questionários e texto da plataforma. & A \\
\hline Yoon; Armour (2017) & $\begin{array}{l}1 \text { Professor formador, } 8 \text { profes- } \\
\text { sores e } 41 \text { alunos }\end{array}$ & Qualitativo/ longitudinal & $\begin{array}{l}\text { Entrevista semiestruturada, Observações, entrevista grupo focal, ques- } \\
\text { tionário e artefatos }\end{array}$ & A \\
\hline Patton; Parker (2017) & 36 professores formadores & Qualitativo/transversal & Entrevistas formai e informais, notas de campo e artefatos & A \\
\hline Eirín-Nemiña (2018) & 7 professores & Qualitativo/ longitudinal & $\begin{array}{l}\text { Notas de campo do observador participante, entrevistas com grupo } \\
\text { focal e artefatos. }\end{array}$ & A \\
\hline Oliver et al. (2018) & $\begin{array}{l}1 \text { professora universitária, } 3 \\
\text { doutorandos e } 1 \text { universitário }\end{array}$ & $\begin{array}{l}\text { Pesquisa-ação partici- } \\
\text { pativa }\end{array}$ & $\begin{array}{l}\text { Notas de campo, discussões após observações, artefatos de professor, } \\
\text { reuniões em grupo e entrevistas individuais. }\end{array}$ & A \\
\hline Luguetti et al. (2018) & 3 professores e 1 amigo crítico & Autoestudo colaborativo & $\begin{array}{l}\text { Notas de campo, entrevistas após observações, artefatos, reuniões } \\
\text { semanais de grupo colaborativo e entrevistas com amigo crítico }\end{array}$ & A \\
\hline Luguetti et al. (2019) & $\begin{array}{l}33 \text { estudantes de graduação e } 4 \\
\text { professores formadores }\end{array}$ & $\begin{array}{l}\text { Pesquisa-ação partici- } \\
\text { pativa }\end{array}$ & $\begin{array}{l}\text { Reuniões semanais em grupo de palestras colaborativas após cada aula, } \\
\text { diários reflexivos dos alunos, observaç̃̃es semanais do professor princi- } \\
\text { pal postadas em rede social e grupos focais de estudantes. }\end{array}$ & A \\
\hline
\end{tabular}

Nota: CASP - Critical Appraisal Skills Programme.

De fato, Deglau e O'Sullivan (2006) apresentam alguns dos motivos manifestados pelos professores para participar de CdP: incentiva os professores a interpretar suas questões educacionais; proporciona aos professores a apropriação das mudanças no currículo, desenvolve um meio de sustentar a interação dos professores e trabalha diretamente nas necessidades dos professores. Uma CdP constitui-se por um grupo de pessoas dispostas a aprender e ensinar por meio da interação e compartilhamento de experiências sobre o tema abordado.

Uma CdP é considerada um ambiente de aprendizagem social em que os participantes se sentem seguros em apresentar suas ideias, seus medos, seus sucessos e fracassos. Desse modo, a troca de experiências e de informações entre os participantes é essencial para que o objetivo em comum seja alcançado. Conforme verificado nos relatos do estudo de Patton e Parker (2017, p. 354, tradução nossa) "se você estivesse em uma instituição e não há muitos professores formadores ao seu redor ou você está em um programa muito pequeno, como desenvolve sua prática?", "Alguém com quem eu poderia conversar como colega". De maneira similar, a importância das trocas de experiências entre os participantes também foi mencionada no estudo de Parker, Patton e Tannehill (2012, p. 316, tradução nossa), pois ela possibilita "captar novas ideias e observar os novos desenvolvimentos que acontecem dentro do assunto em um nível muito prático".

A CdP é projetada para facilitar a mudança das práticas dos professores e são consideradas uma forma especializada de aprendizagem e desenvolvimento profissional, onde os participantes podem trocar informações, sobre diversos aspectos, principalmente a respeito da prática pedagógica. Na última década estudos tem evidenciando que professores conseguiram mudar a sua prática pedagógica através de uma aprendizagem ativa, com a possibilidade de testar novas ideias em seu próprio contexto de ensino (PARKER et al., 2010; GOODYEAR; CASEY; KIRK, 2014; MacPHAIL et al., 2014; PATTON; PARKER, 2017; TANNEHILL; MacPHAIL, 2017).

\section{Engajamento mútuo}

O engajamento mútuo das CdP investigadas ocorreu em diferentes contextos: grupos de professores que trocavam informações por meio de redes sociais (GOODYEAR; CASEY; KIRK, 2014; GOODYEAR; CASEY, 2015; MARTIN, 2017); estágio obrigatório do curso de graduação (GOMES et al., 2014; CARDOSO; BATISTA; GRAÇA, 2016); disciplina teórico-prática do curso de graduação (LUGUETTI et al., 2018); grupo de professores com encontros presenciais (PARKER et al., 2010; PATTON; PARKER; TANNEHILL, 2012; HUNUK; INCE; TANNEHILL, 2013; PATTON; PARKER; PRATT, 2013; PATTON; PARKER, 2017; YOON; ARMOUR, 2017; YOUNG; MacPHAIL, 2015; EIRÍN-NEMIÑA, 2018; OLIVER et al., 2018; LUGUETTI et al., 2019).

Como pode-se observar, das CdP investigadas, apenas três foram criadas no ambiente universitário, gerando a interação 
entre professores formadores e futuros professores. As demais, com exceção ao estudo de Martin (2017), no qual pessoas com diferentes titulações participaram, as CdP foram formadas por professores em atuação. O engajamento mútuo ocorre a partir da interação entre os membros da comunidade, em que a prática liga os participantes entre si, desenvolvendo relações diversas (WENGER, 1998). Conforme trecho de entrevista do estudo de Patton e Parker (2017, p. 355, tradução nossa):

Temos uma profunda amizade em torno do que somos atualmente pensando sobre. É tão divertido conversar porque nos apoiamos. Compartilhamos muito de nossas vidas acadêmicas e eles [outros] não entendem. Eles não entendem suas lutas, suas inseguranças, mas esse grupo entende.

O compartilhamento de informações em ambientes virtuais foram utilizadas como uma forma de diminuir as barreiras geográficas entre os participantes, conforme trecho da entrevista do estudo de Goodyear e Casey (2015, P. 193, tradução nossa): "O Twitter e o Facebook provaram ser uma maneira eficaz de eu me comunicar com os professores esta semana [...] Por exemplo, a Srta. Scholes e a Srta. Collie conversaram comigo na sexta-feira à noite sobre como as aulas foram ministradas.".

O uso das redes sociais para estabelecer relacionamento entre os professores diminui as limitações de distanciamento, de tempo, de recursos e de acesso a conteúdos relevantes e se destacam como uma forma promissora para a criação e sustentação de CdP (GOODYEAR; PARKER; CASEY, 2019). Professores frequentemente usam como fonte de informações diferentes sites de mídia social, como o Facebook, Instagram, Youtube e o Twitter. De fato, estas redes sociais têm se tornado cada vez mais populares para fins de desenvolvimento profissional.

Para que os membros se sintam confiantes e à vontade para compartilhar as suas experiências e conhecimentos com os demais, é necessário que cada participante sinta que está em um ambiente seguro, sem julgamentos, como se observa na declaração de uma professora participante do estudo de Patton e Parker (2017, p. 357, tradução nossa): “O mais significativo foi... criar um ambiente onde eu me sentia seguro para falar sobre coisas [...]". Ainda, a participante investigada, acrescenta "isso foi tão importante para que seja tão seguro para mim poder dizer 'eu não sei o que você quer dizer com isso'". Desse modo, as CdP somente farão sentido para os participantes, quando eles sentirem que as relações transcendem o fator profissional. Conforme o relato de um facilitador do estudo de Patton, Parker e Pratt (2013, p. 447, tradução nossa):

Você tem que criar essa construção social. Quando você deseja criar um relacionamento com alguém, precisa ter um contexto e, geralmente, é social. Se você conhecer alguém fora de um relacionamento profissional, isso lhe dará a oportunidade de ficar muito mais próximo e ser muito mais aberto. As pessoas começam a se abrir. Não há nada como uma cerveja e um ambiente casual que realmente fazem as pessoas falarem. Eles falam sobre si mesmos e sobre coisas pessoais, e isso conecta você ... então você cria essa estrutura social.

As CdP se diferenciam de outras formas de comunidades ou grupos pelo fato de que os membros se engajam de uma maneira partilhada. Em outras palavras, ao participar de uma comunidade, os membros reconhecem que o aprendizado ocorre a partir da troca ou do compartilhamento. Diferente de cursos ou palestras, em que apenas uma pessoa transmite a informação, nas CdP os membros devem estar comprometidos em aprender coletivamente da interação e trocas de experiências (LAVE;
WENGER, 1991).

Ao investigarem durante quinze meses uma CdP com vinte e cinco professores de Educação Física, O'Sullivan e Deglau (2006), destacam que o compromisso e o envolvimento entre os membros foi se modificando ao longo do tempo. Durante o período investigado, observou-se uma mudança no comportamento em relação a responsabilidade que cada membro possui no desenvolvimento dos pares. Assim, se reconhece que os membros de uma CdP não devem estar preocupados apenas com o seu aprendizado, mas sim, com o desenvolvimento de toda a comunidade. Para que os participantes sejam estimulados a colaborar entre si, se faz necessário que a CdP contemple em sua estrutura ao menos um facilitador (CULVER; TRUDEL, 2008).

Dos estudos selecionados, apenas seis tornaram explícito a presença e atuação dos facilitadores. Acredita-se que estes possuem um papel fundamental na sustentação das $\mathrm{CdP}$, conforme o relato de um participante do estudo de Parker et al. (2010, p. 347, tradução nossa): "Sem eles, isso (a CdP) não teria acontecido". De fato, os facilitadores são aqueles que ficam responsáveis, que organizam os encontros, promovem as atividades e otimizam as interações entre os participantes (CALLARY; WERTHNER; TRUDEL, 2013). Conforme o trecho de entrevista de uma participante da CdP retirado do estudo de Parker et al. (2010, p. 348, tradução nossa):

Em vez de achar esse tipo de suporte autoritário, os quatro professores descobriram que aprender com esses "especialistas" contribuiu para a vontade de fazer mudanças. Essa disposição se deve em parte a abordagem dos facilitadores ao desenvolvimento profissional.

De fato, o facilitador de uma CdP de professores, deve auxiliá-los a promover mudanças na prática pedagógica. Para isso, os facilitadores não podem simplesmente oferecer dicas ou conselhos, mas devem, sobretudo, estimular os membros a buscar estratégias apropriadas a partir de problemas encontrados em suas práticas. Assim, o facilitador auxilia os membros da CdP em diferentes processos, como: investigar a própria prática, refletir sobre a sua prática, compartilhar as suas experiências, mantendo um equilíbrio entre o desafio e o auxílio, o que de fato caracteriza a pedagogia da facilitação (POEKERT, 2011).

\section{Repertório compartilhado}

O repertório compartilhado diz respeito a ferramentas, documentos, rotinas e comportamentos desenvolvidos a partir da interação entre os membros das CdP. Este é o principal resultado da participação em CdP. Ao longo do tempo, a comunidade vai se tornando cada vez mais homogênea e os participantes se tornam cada vez mais semelhantes, seja na forma de ensinar, de se expressar, de se vestir, de se organizar, dentre outros elementos (WENGER, 1998). Neste sentido, as CdP investigadas parecem ter cumprido com o objetivo ou com o empreendimento em conjunto.

Através de uma CdP com o auxílio de facilitadores, foi possível verificar que os participantes tiveram sucesso na elaboração de currículo, conforme o relato do participante do estudo de Parker et al. (2010, p. 353, tradução nossa): "Foi um verdadeiro sentimento de realização em nosso trabalho duro; foi simplesmente brilhante que tínhamos conseguido isso e fizemos juntos. Agora, nós compartilhamos com nossos colegas". De forma 
similar, observou-se como um futuro professor qualificou sua prática pedagógica referente aos procedimentos de gestão e instrução, por meio de uma CdP organizada durante o estágio obrigatório, sobre aprender a ensinar Educação Física, conforme trecho de entrevista da professora formadora, do estudo de Cardoso, Batista e Graça (2016, p. 137):

O Henrique (futuro professor) ajustou bem a organização da aula aos alunos que estiveram disponíveis para fazer e atribuiu tarefas aos alunos dispensados. Exemplificou o exercício da chamada para o remate e explicou-o com clareza, focando os pontos críticos. Manteve os alunos sentados enquanto explicava/exemplificava os exercícios, e dispostos em semicírculo para que todos observassem atentamente. Utilizou um tom de voz mais 'amigável' e menos iró nico. Está por isso a fazer grandes progressos.

O uso de diferentes abordagens de ensino a partir de uma CdP foi o objeto de estudo de Hunuk, Ince e Tannehill (2013). Para os autores, durante o desenvolvimento da pesquisa foi possível observar a mudança de uma abordagem centrada no professor para uma abordagem centrada no aluno, conforme trecho de entrevista do facilitador:

Acredito que os professores ficaram impressionados quando viram os diferentes métodos de ensino usados no ambiente escolar, quero dizer na prática. Tivemos a chance de discutir isso em reuniões. Eu gravei alguns exemplos reais da aula para mostrar a eles diferentes metodologias de ensino. A princípio, eles estavam preocupados em como implementá-los, uma ou duas semanas depois eles internalizaram e praticaram em suas próprias escolas. Então eles se tornaram eficientes em usá-los e ficaram impressionados com resultado quando praticaram (HUNUK; INCE; TANNEHILL, 2013, p. 16 , tradução nossa)

O desenvolvimento profissional ou aperfeiçoamento da prática pedagógica de professores em atuação foi evidenciado em seis estudos selecionados. Desse modo, ao participar das CdP os membros parecem ter desenvolvido novas práticas, conforme trecho de entrevista do estudo de Eirín-Nemiña (2018, p. 268, tradução nossa): "Fiquei muito impressionado com a maneira de estruturar o trabalho em sala de aula nesse modelo de ensino, onde a autonomia, a responsabilidade e a cooperação de cada um dos membros do grupo são importantes". Também é possível observar o repertório compartilhado no trecho de entrevista do estudo de Parker, Patton e Tannehill (2012, p. 317, tradução livre): "É algo que você pode levar de volta à sala de aula, usar repetidamente e voltar e atualizar. Algo que você pode usar. Algo em que você pode obter ideias e adaptá-las para se adequar".

As CdP são excelentes contextos para o desenvolvimento profissional, seja qual for o objetivo. O resultado dessa interação, o repertório compartilhado, é evidenciado pela fala de um facilitador do estudo de Parker, Patton, Tannehill (2012, p. 317, tradução nossa): "Tivemos o benefício de compartilhar o que funcionava de outras pessoas. Estávamos procurando soluções de um ser superior ou algo assim, mas na verdade encontramos as soluções dentro de nós mesmos". As trocas de informações e os "ajustes" realizados pelos membros da CdP para que seja produzido o repertório compartilhado é observado no trecho de entrevista do estudo de Patton e Parker (2017, p. 355, tradução nossa): "No final do dia você tem uma mistura mais rica com as ideias de um que equilibra as ideias de outros. O ponto principal para todos foi que precisamos um do outro. Simplesmente eles eram melhores juntos do que separados".

A aprendizagem e o desenvolvimento profissional de professores parece ter sido alcançada através da participação de CdP. De acordo com Lave e Wenger (1991) profissionais de uma mesma função aprendem ou desenvolvem suas práticas compartilhando suas experiências. Esse grupo de pessoas melhora a sua prática partindo das dificuldades encontradas no campo de atuação. Dessa forma, se reconhece que por meio da interação social e dos laços comunitários, os profissionais aprendem novas práticas ou as aprimoram.

Especificamente no âmbito da Educação Física, as CdP são consideradas uma possibilidade eficaz para o desenvolvimento profissional de professores (PATTON; PARKER, 2014). Diante dessa perspectiva de mudança na prática docente e das possibilidades de um melhor desenvolvimento profissional, as CdP permitem que os professores discutam, troquem informações, relatem seus sucessos e fracassos (TANNEHILL; MacPHAIL, 2017).

\section{CONCLUSÃO}

O presente estudo teve como objetivo realizar uma metassíntese a respeito dos fatores que influenciam o desenvolvimento e manutenção das comunidades de prática de professores e futuros professores de Educação Física. Os resultados dos estudos selecionados nos indicam que as comunidades de prática na formação e no desenvolvimento profissional tem origem a partir das necessidades dos interessados.

Assim, as CdP tem origem quando um grupo de pessoas buscam aprender os mesmos assuntos, resolver os mesmos problemas ou se aperfeiçoarem em práticas semelhantes. A manutenção ocorre a partir das trocas de experiências profissionais e das relações pessoais informais. Os encontros foram realizados no formato presencial e por meio de redes sociais. Esses dois ambientes parecem ter sido benéficos para a aprendizagem profissional, pois possibilitaram que os participantes descrevessem suas experiencias. Outro aspecto que contribuiu para a manutenção das comunidades, foram os resultados alcançados. As comunidades de prática investigadas parecem ter satisfeito as necessidades dos participantes. Em suma, as comunidades de prática se apresentam como um excelente contexto de aprendizagem e desenvolvimento profissional. Para que este ambiente colaborativo seja criado e sustentado, o papel do facilitador parece ser fundamental. É ele que tem a função de estimular a interação entre os participantes, organizar os encontros e criar ambientes seguros.

Desse modo, se sugere que as futuras comunidades de prática de professores de Educação Física sejam formadas em diferentes contextos: Projetos de extensão nas universidades, durante os estágios obrigatórios, em escolas, pelas redes sociais e demais contextos informais. Ao destacar a sua função, a sugestão é que o foco das futuras investigações sobre as comunidades de prática, busque compreender a atuação do facilitador.

\section{REFERÊNCIAS}

BARDIN, L. Análise de conteúdo. 3. ed. Lisboa: Edições 70, 2016

BERTRAM, R.; CULVER, D. M.; GILBERT, W. A university sport coach community of practice: Using a value creation framework to explore learning and social interactions. International Journal of Sports Science \& Coaching, Teller Road, v. 12, n. 3, p. 287-302, 2017.

CALLARY, B.; WERTHNER, P.; TRUDEL, P. Exploring coaching actions based on developed values: A case study of a female hockey coach. International Journal of Lifelong Education, Nottingham, v. 32, n. 2, p. 209-29, 2013.

CARDOSO, I.; BATISTA, P.; GRAÇA, A. Narrativas acerca da formação de professores de educação física em contexto de prática supervisionada. Sociologia, Porto, v. 32, p. 125-45, 2016. 
CULVER, D.; TRUDEL, P. Clarifying the concept of communities of practice in sport. International Journal of Sports Science \& Coaching, Teller Road, v. 3 , n. 1, p. 1-10, 2008

DEGLAU, D.; O'SULLIVAN, M. The effects of a long-term professional development program on the beliefs and practices of experienced teachers. Journal of Teaching in Physical Education, Blacksburg, v. 25, n.4, p. 379-76, 2006.

EIRÍN-NEMIÑA, R. Las comunidades de aprendizaje como estrategia de desarrollo profesional de docentes de Educación física. Estudios Pedagógicos, Valdivia, v. 44, n. 1, p. 259-78, 2018

ELLERY, A. E. L.; PONTES, R. J. S.; LOIOLA, F. A. Comunidade de prática enquanto modo coletivo de aprendizagem e desenvolvimento de práticas e saberes na estratégia saúde da família: um estudo teórico. Revista Brasileira em Promoção de Saúde, Fortaleza, v. 25, n. 2, p. 104-12, 2012.

FLORES, P. P.; SILVA CARAÇATO, Y. M.; ANVERSA, A. L. B.; SOLERA, B.; COSTA, L. C. A.; OLIVEIRA, A. A. B.; SOUZA, V. D. F. M. Formação inicial de professores de Educação Física: um olhar para o estágio curricular supervisionado. Caderno de Educação Física e Esporte, v. 17, n. 1, p. 61-68, 2019.

GOMES, P. M. S.; ALVES, M.; QUEIRÓS, P.; BATISTA, P. Learning through practice: a study with physical education pre-service teachers. The Open Sports Sciences Journal, Sharjah, v. 7, n. 1, p. 121-32, 2014.

GOODYEAR, V. A.; CASEY, A. Innovation with change: developing a community of practice to help teachers move beyond the 'honeymoon' of pedagogical renovation. Physical Education and Sport Pedagogy, London, v. 20, n. 2, p. 186-203, 2015.

GOODYEAR, V. A.; CASEY, A.; KIRK, D. Tweet me, message me, like me: Using social media to facilitate pedagogical change within an emerging community of practice. Sport, Education and Society, London, v. 19, n. 7, p. 927-43, 2014.

GOODYEAR, V. A. PARKER, M. CASEY, A. Social media and teacher professional learning communities. Physical Education and Sport Pedagogy, London, v. 24, n. 5 , p. $421-33,2019$

HUNUK, D.; INCE, M. L.; TANNEHILL, D. Developing teachers' health-related fitness knowledge through a community of practice: Impact on student learning. European Physical Education Review, Chester, v. 19, n. 1, p. 3-20, 2013.

HUNUK, D; TANNEHILL, D; INCE, M. L. Interaction patterns of physical education teachers in a professional learning community. Physical Education and Sport Pedagogy, London, v. 24, n. 3, p. 301-17, 2019.

LAVE, J.; WENGER, E. Situated learning: Legitimate peripheral participation. Cambridge: Cambridge University Press, 1991.

LUGUETTI, C.; ARANDA, R.; NUÑEZ ENRIQUEZ, O.; OLIVER., K. L. Developing teachers' pedagogical identities through a community of practice: Learning to sustain the use of a student-centered inquiry as curriculum approach. Sport, Education and Society, London, v. 24, n. 8, p. 1-12, 2018

LUGUETTI, C.; GOODYEAR, V. A. ANDRÉ, M. H. 'That is like a 24 hours-day tournament!': using social media to further an authentic sport experience within sport education. Sport, Education and Society, London, v. 24, n. 1, p. 78-91, 2019

MacPHAIL, A.; PATTON, K.; PARKER, M.; TANNEHILL, D. Leading by example: Teacher educators' professional learning through communities of practice. Quest, Cambridge, v. 66, n. 1, p. 39-56, 2014.

MARTIN, P. D. V. Multiscopic, análisis de una Comunidad de Práctica Virtual en Educación Física. Sportis, Valladolid, v. 3, n. 2, p. 388-403, 2017.

OLIVER, K. L.; LUGUETTI, C.; ARANDA, R.; NUÑEZ ENRIQUEZ, O.; RODRIGUEZ, A. 'Where do I go from here?': Learning to become activist teachers through a community of practice. Physical Education and Sport Pedagogy, London, v. 23, n. 2, p. 150-65, 2018

PARKER, M.; PATTON, K.; MADDEN, M.; SINCLAIR, C. From committee to community: The development and maintenance of a community of practice. Journal of Teaching in Physical Education, Blacksburg, v. 29, n. 4, p. 337-57, 2010.

PARKER M. PATTON, K. TANNEHILL, D. Mapping the landscape of communities of practice as professional development in Irish physical education. Irish Educational Studies, Dublin, v. 31, n. 3, p. 311-27, 2012.

PATTON, K.; PARKER, M. Moving from 'things to do on Monday'to student learning: physical education professional development facilitators' views of success. Physical Education and Sport Pedagogy, London, v. 19, n. 1, p. 60 75, 2014.

PATTON, K.; PARKER, M. Teacher education communities of practice: More than a culture of collaboration. Teaching and Teacher Education, Amsterdam, v. 67, p. 351-60, 2017.
PATTON, K: PARKER, M; PRATT, E. Meaningful learning in professional development: Teaching without telling. Journal of Teaching in Physical Education, Blacksburg, v. 32, n. 4, p. 441-59, 2013

POEKERT, P. The pedagogy of facilitation: Teacher inquiry as professional development in a Florida elementary school. Professional Development in Education, Leeds, v. 37, n. 1, p. 19-38, 2011.

SANDELOWSKI, M.; BARROSO, J. Handbook for synthesizing qualitative research. Berlim: Springer, 2006.

TANNEHILL, D.; MacPHAIL, A. Teacher empowerment through engagement in a learning community in Ireland: Working across disadvantaged schools. Professional Development in Education, Leeds, v. 43, n. 3, p. 334-52, 2017.

VYGOTSKY, L. S. A formação social da mente: o desenvolvimento dos processos psicológicos superiores. São Paulo: Martins Fontes, 1991.

WENGER, E. Communities of practice: learning, meaning, and identity. New York: Cambridge University Press, 1998.

YOON, K.; ARMOUR, K. M. Mapping physical education teachers' professional learning and impacts on pupil learning in a community of practice in South Korea. Physical Education and Sport Pedagogy, London, v. 22, n. 4 , p. 427-44, 2017

YOUNG, A.; MacPHAIL, A. 'Standing on the periphery' Cooperating teachers' perceptions and responses to the role of supervision. European Physical Education Review, Chester, v. 21, n. 2, p. 222-37, 2015

ZIMMER, L. Qualitative meta-synthesis: a question of dialoguing with texts. Journal of Advanced Nursing, Hull, v. 53, n. 3, p. 311-8, 2006.

\section{CONFLITO DE INTERESSE}

Os autores do estudo declaram não haver conflito de interesses.

\section{FINANCIAMENTO}

Este estudo teve apoio financeiro do Programa de Bolsas Universitárias de Santa Catarina - Uniedu.

\section{ORCID E E-MAIL DOS AUTORES}

Leonardo Ristow (Autor Correspondente)

ORCID: 0000-0002-2829-7737.

E-mail: leonardoristow@live.com

Ana Flávia Backes

ORCID: 0000-0002-3949-8809.

E-mail: anafbackes@hotmail.com

Daniela Bianchessi

ORCID: 0000-0002-8484-722X

E-mail: daniela.bianchessi@unifebe.edu.br

Vinicius Zeilmann Brasil

ORCID: 0000-0003-0036-494X.

E-mail:vzbrasil@hotmail.com

Rodolfo Silva da Rosa

ORCID: 0000-0002-5353-1820.

E-mail: rodolfodarosa@yahoo.com.br

Jessica Cardoso

ORCID: 0000-0002-6910-3767.

E-mail: jessicacardooso@outlook.com

Valmor Ramos

ORCID: 0000-0002-1659-5702.

E-mail:valmor.ramos@udesc.br 